\title{
SOME RESULTS ON PRINCIPAL EIGENVALUES FOR PERIODIC PARABOLIC PROBLEMS WITH WEIGHT
}

\author{
U. KAUFMANN
}

Let $\Omega \subset \mathbb{R}^{N}$ be a bounded domain. We study existence and uniqueness of principal eigenvalues for the Dirichlet periodic parabolic problem with weight $L u=\lambda m u$ in $\Omega \times \mathbb{R}$ when the independent coefficient of the differential operator $L$ is not necessarily positive.

\section{INTRODUCTION}

Let $\Omega \subset \mathbb{R}^{N}, N \geqslant 2$, be a bounded domain. For $T>0$ and $1 \leqslant p, q \leqslant \infty$, let $L^{p}\left(L^{q}\right)$ be the Banach space of $T$-periodic functions $f$ on $\Omega \times \mathbb{R}$ (that is, satisfying $f(x, t)=f(x, t+T)$ almost everywhere $(x, t))$ with \|\|$f(\cdot, t)\left\|_{L^{q}(\Omega)}\right\|_{L^{p}(0, T)}<\infty$. Let $L_{T}^{p}$ be the Banach space of $T$-periodic functions $f: \Omega \times \mathbb{R} \rightarrow \mathbb{R}$ such that $f_{\mid \Omega \times(0, T)}$ $\in L^{p}(\Omega \times(0, T))$, equipped with its natural norm $\|f\|_{L_{T}^{p}}=\left\|f_{\mid \Omega \times(0, T)}\right\|_{L^{p}(\Omega \times(0, T)}$. We fix from now on $v, s \in(1, \infty]$ such that $(N / 2 v)+1 / s<1$, with $s \geqslant 2$. Let $\left\{a_{i, j}\right\},\left\{b_{j}\right\}$, $1 \leqslant i, j \leqslant N$, be two families of $T$-periodic functions satisfying $a_{i, j} \in L_{T}^{\infty}, a_{i, j}=a_{j, i}$ for $1 \leqslant i, j \leqslant N$ and $b_{j} \in L^{\infty}\left(L^{2 v}\right)$. Assume that $\sum_{i, j} a_{i, j}(x, t) \xi_{i} \xi_{j} \geqslant \alpha_{0}|\xi|^{2}$ for some $\alpha_{0}>0$ and all $(x, t) \in \Omega \times \mathbb{R}, \xi \in \mathbb{R}^{N}$. Let $a_{0}(x, t)$ be a $T$-periodic function, and let $L$ be the parabolic operator given by

$$
L u=u_{t}-\sum_{i} \frac{\partial}{\partial x_{i}} \sum_{j} a_{i, j} \frac{\partial}{\partial x_{j}} u+\sum_{i} b_{i} \frac{\partial}{\partial x_{i}} u+a_{0} u
$$

The existence of principal eigenvalues (that is, eigenvalues with positive eigenfunctions associated) for the periodic parabolic problem with $T$-periodic weight function $m: \Omega \times \mathbb{R} \rightarrow \mathbb{R}$

$$
\begin{cases}L u=\lambda m u & \text { in } \Omega \times \mathbb{R} \\ u=0 & \text { on } \partial \Omega \times \mathbb{R} \\ u T \text {-periodic } & \end{cases}
$$

\section{Received 2nd October, 2002}

Partially supported by UAM/BSCH (Spain), CONICET, Secyt-UNC and Agencia Cba. Ciencia (Argentina). The author would kindly like to dedicate this work to his teacher and friend Tomás Godoy. He would also like to thank Professor J. Hernández for his fruitful suggestions.

Copyright Clearance Centre, Inc. Serial-fee code: 0004-9727/03 \$A2.00+0.00. 
has been widely studied. For applications we refer to [10]. In [2], Beltramo and Hess proved, for $C^{2+\theta}$ bounded domains, weights $m \in C^{\theta, \theta / 2}(\bar{\Omega} \times \mathbb{R})$ and operators in nondivergence form, that if

$$
P(m):=\int_{0}^{T} \underset{x \in \Omega}{\operatorname{ess} \sup } m(x, t) d t
$$

then $P(m)>0$ is a necessary and sufficient condition on $m$ for the existence, uniqueness and (algebraic) simplicity of a positive principal eigenvalue. Later on there were many extensions of their results, weakening the regularity assumptions in the weight and the domain and also allowing more general boundary conditions (for example, $[1,9,3,4,8]$ and the references therein). In all these works it is always assumed that the independent coefficient $a_{0}$ is nonnegative. On the other hand, the analogous elliptic problem was treated in [6] for operators with an independent coefficient which changes sign in $\Omega$, using as main tools the Krein-Rutman theorem as well as the variational characterisation of the first eigenvalue.

Our aim in this paper is to study problem (1.1) when $a_{0}$ changes sign in $\Omega \times \mathbb{R}$. Of course, we do not have the variational characterisation here and so we cannot follow the approach in [6]. We shall follow instead the main ideas used to treat (1.1) when $a_{0} \geqslant 0$, namely we shall study the family of auxiliary eigenvalue problems

$$
\begin{cases}L u=\lambda m u+\mu_{m, L}(\lambda) u & \text { in } \Omega \times \mathbb{R} \\ u=0 & \text { on } \partial \Omega \times \mathbb{R} \\ u T \text {-periodic } & \end{cases}
$$

where, for each $\lambda \in \mathbb{R}, \mu_{m, L}(\lambda)$ is defined as the only real number such that (1.3) has a positive solution.

Let $L_{0}$ be the operator defined by $L_{0}=L+a_{0}^{-}$. Let us denote by $\lambda_{1}(L, m)$ (or $\lambda_{1}(m)$ if no confusion arises) the positive principal eigenvalue for (1.1). We shall prove that if $a_{0}^{-}$is small enough, namely if $\lambda_{1}\left(L_{0}, a_{0}^{-}\right)>1$, then $P(m)>0$ is still necessary and sufficient for the existence of a unique positive principal eigenvalue. When $\lambda_{1}\left(L_{0}, a_{0}^{-}\right)=1$, it turns out that the above condition is necessary but no longer sufficient. We shall give a sufficient condition for this case. Finally, if $\lambda_{1}\left(L_{0}, a_{0}^{-}\right)<1$ we shall show that there can exist one or two positive principal eigenvalues and that $P(m)>0$ is not even necessary in this case.

\section{Preliminaries}

For $f: \Omega \times \mathbb{R} \rightarrow \mathbb{R}$, let $f^{+}=\max (f, 0)$ and $f^{-}=\max (-f, 0)$. We shall assume throughout the paper that $a_{0}$ and $m$ satisfy the regularity conditions

$$
a_{0}^{+}, m^{+} \in L^{s}\left(L^{v}\right), \quad a_{0}^{-}, m^{-} \in L_{T}^{\infty}
$$


REMARK 2.1. Let us define $\mu$ when $a_{0}$ changes sign in $\Omega \times \mathbb{R}$ (if no confusion arises we shall write $\mu_{m}(\lambda)$ or simply $\mu(\lambda)$ instead of $\left.\mu_{m, L}(\lambda)\right)$. Let $\lambda \geqslant 0$. Adding $k u, k$ constant, to both sides of (1.3), the problem $L u=\lambda m u+\mu(\lambda) u$ is equivalent to

$$
(L+(k-\lambda m)) u=(\mu(\lambda)+k) u
$$

Now, for $k$ large enough the operator $(L+(k-\lambda m))^{-1}: L^{r}\left(L^{p}\right) \rightarrow L^{r}\left(L^{p}\right)$ is compact, positive and irreducible (see [8, Proposition 2.4 and Lemma 2.6]), where $r$ and $p$ are given by [8, Lemma 2.1]. Let us denote by $\rho(T)$ the spectral radius of $T$. Thus, KreinRutman's theorem (for example, [5, Theorem 12.3]) says that $\rho\left((L+(k-\lambda m))^{-1}\right)$ is positive, is an algebraically simple eigenvalue of $(L+(k-\lambda m))^{-1}$ and that it is the only eigenvalue with positive eigenfunctions associated. Taking into account this and (2.1), we define $\mu(\lambda)$ by

$$
\mu(\lambda)=\frac{1}{\rho\left((L+(k-\lambda m))^{-1}\right)}-k
$$

It is easy to see that $\mu(\lambda)$ does not depend on the choice of $k$. For $\lambda<0$ we set $\mu_{m}(\lambda)=\mu_{-m}(-\lambda)$. Note that $\mu(\lambda)$ can be characterised as the unique real number such that (1.3) has a positive solution.

REMARK 2.2. It is known that $\mu$ es continuous and concave (see [8, Lemma 3.2]). Moreover, $\lim _{\lambda \rightarrow \infty} \mu(\lambda)=-\infty$ if and only if $P(m)>0$ (see [8, Lemma 3.5 and Theorem 3.6]). For weights $m \in L_{T}^{\infty}$, these results can also be found in [4, Proposition 3.2].

In the known case, that is, $a_{0}^{-} \equiv 0$, it also holds that $\mu(0)>0$, and thus this and the properties of $\mu$ listed above give the existence of a (unique) positive principal eigenvalue for $(1.1)$ if and only if $P(m)>0$. However, this property is not true in general any more when $a_{0}$ changes sign. In fact, $\mu(0)$ can be positive, zero or negative as we shall see in the next section.

REMARK 2.3. Let us recall the definition of $\mu$ when $a_{0}$ is nonnegative. Assume here that $a_{0} \geqslant 0$. Adding $\lambda k u$ to both sides of (1.3) and reasoning as in Remark 2.1 we have that for $k$ large enough (1.3) is equivalent to

$$
\frac{1}{\mu(\lambda)+\lambda k} u=(L+\lambda(k-m))^{-1} u
$$

and so by the Krein-Rutman theorem we may define $\mu$ by

$$
\rho\left((L+\lambda(k-m))^{-1}\right)=\frac{1}{\mu(\lambda)+\lambda k}
$$

(Note that in this case $\mu(0)=1 /\left(\rho\left(L^{-1}\right)\right)>0$ since Krein-Rutman applies to $L^{-1}$ because $a_{0} \geqslant 0$.) In particular, for $\lambda=\lambda_{1}(m)$ we have

$$
\rho\left(\left(L+\lambda_{1}(m)(k-m)\right)^{-1}\right)=\frac{1}{\lambda_{1}(m) k}
$$




\section{Main Results}

In a similar way to [6], we consider separately three cases:

Case I $\lambda_{1}\left(L_{0}, a_{0}^{-}\right)>1$.

Case II $\quad \lambda_{1}\left(L_{0}, a_{0}^{-}\right)=1$.

Case III $\lambda_{1}\left(L_{0}, a_{0}^{-}\right)<1$.

We shall need the following corollary of Krein-Rutman's theorem (see [5, Corollary 12.4]).

Lemma 3.1. Let $T$ be a linear, positive, compact and irreducible operator. Consider the equation

$$
\lambda x-T x=y
$$

with $y \geqslant 0$. Then,

(i) The equation (3.1) has no positive solution for $\lambda<\rho(T)$.

(ii) The equation (3.1) has no solution for $\lambda=\rho(T)$.

\section{THEOREM 3.2 .}

(i) Suppose $\lambda_{1}\left(L_{0}, a_{0}^{-}\right)>1$. Then, $P(m)>0$ is necessary and sufficient for the existence of a positive principal eigenvalue $\lambda_{1}(m)$ for (1.1). Moreover, $\lambda_{1}(m)$ is unique and simple.

(ii1) Suppose $\lambda_{1}\left(L_{0}, a_{0}^{-}\right)=1$. Then, $P(m)>0$ is necessary but not sufficient for the existence of a positive principal eigenvalue for (1.1).

(ii2) The conditions $P(m)>0$ and

$$
\frac{a_{0}^{-}}{\lambda_{1}\left(L_{0}, m^{+}\right)} \leqslant m^{-} \quad \text { almost everywhere }(x, t)
$$

are sufficient for the existence of a positive principal eigenvalue $\lambda_{1}(m)$. Moreover, $\lambda_{1}(m)$ is simple, is the only nonzero principal eigenvalue for (1.1) and $\lambda_{1}\left(L_{0}, m^{+}\right) \leqslant \lambda_{1}(m)<\lambda_{1}\left(L_{0}, m\right)$.

PRoof: We shall prove first that Case I occurs if and only if $\mu_{m, L}(0)>0$ and thus (i) shall follow from Remark 2.2. Assume first $\mu_{m, L}(0)>0$. We have $L u=\lambda m u+\mu_{m, L}(\lambda) u$ for all $\lambda$, with $u>0$. In particular, for $\lambda=0$ we get $L u=\mu_{m, L}(0) u>0$. Then, since $L_{0}=L+a_{0}^{-}$, adding $k u$ to both sides with $k$ large enough and taking into account Remark 2.3 we have

$$
\frac{1}{k} u>\left(L_{0}+1 .\left(k-a_{0}^{-}\right)\right)^{-1} u:=T u
$$

where $T$ satisfies the hypothesis of Lemma 3.1. Now, suppose $1=\lambda_{1}\left(L_{0}, a_{0}^{-}\right)$. Then, from (2.3) we have $\rho(T)=1 / k$ and thus (3.3) implies that $\rho(T) u-T u>0$ which contradicts Lemma 3.1 (ii). Stappose $1>\lambda_{1}\left(L_{0}, a_{0}^{-}\right)$. Then $\mu_{a_{0}^{-}, L_{0}}(1)<0$ and so from (2.2) we derive 
$\lambda^{*}:=1 / k<1 /\left(k+\mu_{a_{0}^{-}, L_{0}}(1)\right)=\rho(T)$. Thus, it follows from (3.3) that $\lambda^{*} u-T u>0$ with $\lambda^{*}<\rho(T)$ and $u>0$, in contradiction with Lemma 3.1 (i). So we must have $1<\lambda_{1}\left(L_{0}, a_{0}^{-}\right)$.

Assume now that $\lambda_{1}\left(L_{0}, a_{0}^{-}\right)>1$. We have $L u=\mu_{m, L}(0) u$ with $u>0$. If $\mu_{m, L}(0)$ $=0$, we get $L_{0} u=a_{0}^{-} u, u>0$. Thus, $1=\lambda_{1}\left(L_{0}, a_{0}^{-}\right)>1$. Contradiction. Suppose $\mu_{m, L}(0)<0$. Then we obtain $L_{0} u-1 . a_{0}^{-} u<0$. But if $1<\lambda_{1}\left(L_{0}, a_{0}^{-}\right)$[8, Theorem 3.10] says that $u<0$. Contradiction. Thus, $\mu_{m, L}(0)>0$.

Let us prove (ii1). Observe that $\lambda_{1}\left(L_{0}, a_{0}^{-}\right)=1$ if and only if $\mu_{m, L}(0)=0$. Indeed, suppose first $\lambda_{1}\left(L_{0}, a_{0}^{-}\right)=1$. Then there exists $v>0$ such that $L_{0} v=a_{0}^{-} v$ and so $L v=0=0 . v$. But $\mu_{m, L}(0)$ is the only number with such property, thus we must have $\mu_{m, L}(0)=0$. Conversely, if $\mu_{m, L}(0)=0$ we have $L u=\mu_{m, L}(0) u=0$ with $u$ positive and so $L_{0} u=a_{0}^{-} u, u>0$. Thus, $\lambda_{1}\left(L_{0}, a_{0}^{-}\right)=1$. So, the necessity follows as in (i). To prove the remaining assertion, consider the weight $m \equiv m^{+}$. Clearly $P\left(m^{+}\right)>0$. Suppose there exists $\lambda_{1}\left(m^{+}\right)$. Then $\mu_{m^{+}, L}(\lambda) \geqslant 0$ for all $0<\lambda<\lambda_{1}\left(m^{+}\right)$. Thus, for such $\lambda$ we have $L u \geqslant \lambda m^{+} u>0$ with $u>0$, but then as in the beginning of the proof of (i) we get $\lambda_{1}\left(L_{0}, a_{0}^{-}\right)>1$. Contradiction.

We prove (ii2). We claim that (3.2) implies $\mu_{m, L}\left(\lambda_{1}\left(L_{0}, m^{+}\right)\right) \geqslant 0$. Indeed, if not, then $L u<\lambda_{1}\left(L_{0}, m^{+}\right) m u$ for some positive $u$. So we have

$$
L_{0} u<\left(a_{0}^{-}-\lambda_{1}\left(L_{0}, m^{+}\right) m^{-}\right) u+\lambda_{1}\left(L_{0}, m^{+}\right) m^{+} u
$$

and then (3.2) implies $L_{0} u<\lambda_{1}\left(L_{0}, m^{+}\right) m^{+} u$. Now, as in (i) we get

$$
\frac{1}{\lambda_{1}\left(L_{0}, m^{+}\right) k} u<\left(L_{0}+\lambda_{1}\left(L_{0}, m^{+}\right)\left(k-m^{+}\right)\right)^{-1} u=: T u
$$

for some $k$ large enough and thus (2.3) gives $\rho(T) u<T u$. So $\rho(T)(-u)-T(-u)>0$, in contradiction with Lemma 3.1 (ii). Thus, $\mu_{m, L}\left(\lambda_{1}\left(L_{0}, m^{+}\right)\right) \geqslant 0$ and then, since $P(m)>0$, the continuity of $\mu_{m, L}$ gives some $\lambda \geqslant \lambda_{1}\left(L_{0}, m^{+}\right)$such that $\mu_{m, L}(\lambda)=0$. Moreover, since $\lambda=\lambda_{1}\left(L_{0}, m+\left(a_{0}^{-}\right) / \lambda\right)$, [8, Proposition 3.1] implies $\lambda<\lambda_{1}\left(L_{0}, m\right)$. The uniqueness of the principal eigenvalue follows from the concavity of $\mu_{m, L}$ and the fact that $\mu_{m, L}(0)=0$.

Let us note that in the known case, that is, $a_{0} \geqslant 0,(3.2)$ is always satisfied and the condition in (ii2) reduces to $P(m)>0$, which in that case is also necessary. Note also that 0 is a principal eigenvalue for (1.1) if and only if $\lambda_{1}\left(L_{0}, a_{0}^{-}\right)=1$.

REMARK 3.3. Let $N(m)=\int_{0}^{T} \underset{x \in \Omega}{\operatorname{essinf}} m(x, t) d t$ and suppose $\lambda_{1}\left(L_{0}, a_{0}^{-}\right)>1$. Since $\mu_{m}(\lambda)=\mu_{-m}(-\lambda), N(m)<0$ is necessary and sufficient for the existence of a (unique) negative principal eigenvalue $\lambda_{-1}(m)$ for (1.1). On the other hand, if $\lambda_{1}\left(L_{0}, a_{0}^{-}\right)=1$, reasoning as above we can see that $N(m)<0$ is necessary (but not sufficient) for the existence of a negative principal eigenvalue. Moreover, if $\lambda<0$, writing $L u$ $=(-\lambda)(-m) u$ it can be proved that $\left(a_{0}^{-}\right) /\left(\lambda_{1}\left(L_{0}, m^{-}\right)\right) \leqslant m^{+}$almost everywhere $(x, t)$ 
implies $\mu_{m, L}\left(-\lambda_{1}\left(L_{0}, m^{-}\right)\right) \geqslant 0$ and so $N(m)<0$ and $\left(a_{0}^{-}\right) /\left(\lambda_{1}\left(L_{0}, m^{-}\right)\right) \leqslant m^{+}$almost everywhere $(x, t)$ are sufficient for the existence of a negative (unique) principal eigenvalue $\lambda_{-1}(m)$.

In Case III there can be one, two or no positive principal eigenvalues. Although we cannot give a precise answer for each $m$ (even in the elliptic case this does not seem to be possible, see [6, Section 2.3]), we can still give sufficient conditions on $m$ for the existence of one or two positive principal eigenvalues.

Corollary 3.4. Suppose $\lambda_{1}\left(L_{0}, a_{0}^{-}\right)<1$. Then, (3.2) is sufficient for the existence of a positive principal eigenvalue for ((1.1). Moreover,

(i1) If $P(m)>0$ and $\left(a_{0}^{-}\right) /\left(\lambda_{1}\left(L_{0}, m^{+}\right)\right)<m^{-}$in a set of positive measure, then there exist two positive principal eigenvalues $\lambda_{1}(m)$ and $\bar{\lambda}_{1}(m)$. These eigenvalues are simple, are the only principal eigenvalues for (1.1) and $\lambda_{1}(m)<\lambda_{1}\left(L_{0}, m^{+}\right)<\bar{\lambda}_{1}(m)$.

(i2) If $P(m)>0$ and $\left(a_{0}^{-}\right) /\left(\lambda_{1}\left(L_{0}, m^{+}\right)\right)=m^{-}$almost everywhere $(x, t)$, then $\lambda_{1}\left(L_{0}, m^{+}\right)$is a positive principal eigenvalue. This eigenvalue is simple, and if $m=m(t)$, is the only principal eigenvalue for (1.1).

(ii) If $P(m) \leqslant 0$, then there exists a positive principal eigenvalue $\lambda_{1}(m)$. This eigenvalue is simple, is the only principal eigenvalue for (1.1) and $\lambda_{1}(m)$ $\leqslant \lambda_{1}\left(L_{0}, m^{+}\right)$.

Proof: As a consequence of the proof of Theorem 3.2 we see that $\lambda_{1}\left(L_{0}, a_{0}^{-}\right)<1$ if and only if $\mu_{m, L}(0)<0$. On the other side, as in Theorem 3.2 (ii2) we have $\mu_{m, L}\left(\lambda_{1}\left(L_{0}, m^{+}\right)\right) \geqslant 0$. Moreover, it is easy to check that $\mu_{m, L}\left(\lambda_{1}\left(L_{0}, m^{+}\right)\right)=0$ if and only if $\left(a_{0}^{-}\right) /\left(\lambda_{1}\left(L_{0}, m^{+}\right)\right) \equiv m^{-}$and then in this case $\lambda_{1}\left(L_{0}, m^{+}\right)$is a positive principal eigenvalue for (1.1). Thus, (i1), (ii) and the first part of (i2) follow as before. Suppose now $m=m(t)$. Let $m_{s}=m+\left(a_{0}^{-}\right) / s$ and consider the problem $L_{0} u=\lambda m_{s} u+\mu_{s}(\lambda) u$, where $\mu_{s}=\mu_{m_{s}, L_{0}}$. Clearly $s$ is a principal eigenvalue for (1.1) if and only if $\mu_{s}(s)=0$. Without loss of generality we assume $s>0$. Since $m$ does not depend on $x$, taking into account [10, Lemma 15.3] and reasoning as in [4, Lemma 5.4] we can prove that $\mu_{s}(\lambda)=\mu_{s}(0)-(\lambda / T) P\left(m_{s}\right)$ for all $\lambda>0$. Let $\lambda^{*}=\lambda_{1}\left(L_{0}, m^{+}\right)$. Note that by Remark 2.3 we have that $\mu_{s}(0)$ is the same for all s. Moreover, since $\mu_{\lambda^{*}}\left(\lambda^{*}\right)=0$ we obtain $\mu_{s}(0)=\left(\lambda^{*} / T\right) P\left(m_{\lambda^{*}}\right)$. Assume now there exists $s>0$ such that $\mu_{s}(s)=0$. Then we get $\lambda^{*} P\left(m_{\lambda^{*}}\right)=s P\left(m_{s}\right)$, and a computation shows that this implies $\left(\lambda^{*}-s\right) P(m)=0$. Since $P(m)>0$, we must have $\lambda^{*}=s$ and thus the uniqueness is proved.

Let $M_{0}:=\{(x, t) \in \Omega \times \mathbb{R}: m(x, t)=0\}$. Complementing Corollary 3.4 (ii) we have.

Proposition 3.5. Suppose $\lambda_{1}\left(L_{0}, a_{0}^{-}\right)<1, m^{+} \equiv 0$. Assume that $a_{0}^{-} \chi_{M_{0}}=0$ almost everywhere or $\lambda_{1}\left(L_{0}, a_{0}^{-} \chi_{M_{0}}\right) \geqslant 1$, and $\left(a_{0}^{-}\right) /\left(m^{-}\right) \in L^{\infty}\left(V-M_{0}\right)$ where $V$ is a neighbourhood of $M_{0}$. Then, there exists a positive principal eigenvalue $\lambda_{1}(m)$. Moreover, $\lambda_{1}(m)$ is simple and is the only principal eigenvalue for (1.1). 
Proof: Suppose it does not exist $\lambda>0$ such that $\mu_{m, L}(\lambda)=0$. Then, since $\mu_{m, L}(0)<0$ we have $\mu_{m, L}(\lambda)<0$ for all $\lambda>0$. So, $L u<\lambda m u=-\lambda m^{-} u$ for some $u>0$ and thus

$$
L_{0} u<a_{0}^{-} \chi_{M_{0}} u+\left(a_{0}^{-} \chi_{\{m<0\}}-\lambda m^{-}\right) u
$$

for all $\lambda>0$. On the other hand, there exists $\lambda^{*}$ large enough (not depending on $(x, t)$ ) such that $a_{0}^{-} \chi_{\{m<0\}}-\lambda^{*} m^{-} \leqslant 0$ almost everywhere $(x, t) \in \Omega \times \mathbb{R}$ with strict inequality in a set of positive measure. So, (3.4) gives $L_{0} u<a_{0}^{-} \chi_{M_{0}} u$ with $u>0$ and therefore reasoning as in the proof of Theorem 3.2 we get $1>\lambda_{1}\left(L_{0}, a_{0}^{-} \chi_{M_{0}}\right)$, while if $a_{0}^{-} \chi_{M_{0}}=0$ almost everywhere we obtain a contradiction with the positivity of $L_{0}^{-1}$. Thus, the existence of $\lambda_{1}(m)$ is proved. The uniqueness of the principal eigenvalue follows from the concavity of $\mu_{m, L}$ and the fact that $P(m) \leqslant 0$ and $\mu_{m, L}(0)<0$.

Note that in Case III the weight $m \equiv-m^{-}$possesses a positive principal eigenvalue in contrary to what occurs in cases I and II, and that in cases II and III the weight $m \equiv m^{+}$does not have a positive principal eigenvalue in contrary to what occurs in Case I.

REMARK 3.6. Let us conclude with some comments on the case of a general operator. Let us consider

$$
L u=u_{t}-\sum a_{i, j} \frac{\partial^{2} u}{\partial x_{i} \partial x_{j}}+\sum b_{i} \frac{\partial u}{\partial x_{i}}+a_{0} u .
$$

We assume that $\Omega \subset \mathbb{R}^{N}$ is a $C^{2+\theta}$ bounded domain and that $a_{i, j}, b_{i}, a_{0}, m \in C_{T}^{\theta, \theta / 2}(\bar{\Omega} \times \mathbb{R})$. Under these hypothesis, it can be proved in the same way as we did all the results of the three considered cases, in the framework of classical solutions and using the classical version of the Krein-Rutman theorem since all the results we have used are valid with the above hypothesis (see for example, [10]).

Of course, it would be nice to weaken some of the regularity assumptions, especially on the weight. However, there is a problem. The proof given by Hess of the fact that $P(m)>0$ implies $\mu(\lambda) \rightarrow-\infty$ when $\lambda \rightarrow \infty$ (and so the existence of a positive principal eigenvalue when $a_{0} \geqslant 0$ ) depends on the continuity of $m$ and cannot be extended to a noncontinuous weight (see [10, Lemma 15.4], and also [2, 11]). And the alternative proof that it is known uses the weak form of the equation with a suitable test function, and so, or the coefficients $a_{i j}$ are assumed to be $C_{T}^{1}(\bar{\Omega} \times \mathbb{R})$ and then the operator can be rewritten in divergence form (for example, $[9,7]$ ), or the problem starts with an operator in divergence form (for example $[3,4,8]$ ). We suppose that this should be true for a general operator and $m$ not continuous, but we do not know the proof.

\section{REFERENCES}

[1] A. Beltramo, Über den Hauptteigenwert von peridish-parabolischen Differiantialoperatoren, Ph.D. Thesis (Univ. of Zurich, Zurich, 1984). 
[2] A. Beltramo and P. Hess, 'On the principal eigenvalue of a periodic-parabolic operator', Comm. Partial Differential Equations 9 (1984), 919-941.

[3] D. Daners, 'Periodic-parabolic eigenvalue problems with indefinite weight functions', Arch. Math. 68 (1997), 388-397.

[4] D. Daners, 'Existence and perturbation of principal eigenvalues for a periodic-parabolic problem', Electron. J. Differ. Equ. Conf. 5 (2000), 51-67.

[5] D. Daners and P. Koch-Medina, Abstract evolution equations, periodic problems and applications, Pitman Research Notes in Mathematics 279 (Longman Scientific and J. Wiley \& Sons, Harlow and New York, 1992).

[6] J. Fleckinger, J. Hernández and F. de Thélin, 'Existence of multiple principal eigenvalues for some indefinite linear eigenvalue problems', (Preprint).

[7] T. Godoy, A. Guerin and S. Paczka, 'On positive solutions of some periodic parabolic eigenvalue problem with a weight function', Rend. Sem. Mat. Univ. Padova 101 (1999), $1-17$.

[8] T. Godoy and U. Kaufmann, 'On principal eigenvalues for periodic parabolic problems with optimal condition on the weight function', J. Math. Anal. Appl. 262 (2001), 208-220.

[9] T. Godoy, E. Lami Dozo and S. Paczka, 'The periodic parabolic eigenvalue problem with $L^{\infty}$ weight', Math. Scand. 81 (1997), 20-34.

[10] P. Hess, Periodic-parabolic boundary value problems and positivity, Pitmans Research in Mathematics 2471992 (Longman Scientific and J. Wiley \& Sons, Harlow and New York).

[11] P. Hess, On positive solutions of semilinear periodic-parabolic problems, Lecture Notes in Math. 1076 (Springer-Verlag, Berlin, Heidelberg, New York), pp. 101-114.

FaMAF

Universidad Nacional de Córdoba

Ciudad Universitaria

(5000) Córdoba

Argentina

e-mail: kaufmann@mate.uncor.edu 